\title{
Preferential droplet vaporization of a 24-component mixture under different ambient temperature, pressure and force convection
}

\author{
Lei Luo ${ }^{1,2,3}$, Yu Cheng Liu*1,2,3 \\ ${ }^{1}$ Center for Combustion Energy, Tsinghua University, Beijing, China \\ ${ }^{2}$ Department of Energy and Power Engineering, Tsinghua University, Beijing, China \\ ${ }^{3}$ Key Laboratory for Thermal Science and Power Engineering of MOE, Tsinghua University, \\ Beijing, China \\ *Corresponding author email: ycliu7@mail.tsinghua.edu.cn
}

\begin{abstract}
Petroleum-derived fuels like gasoline, kerosene, and diesel contain hundreds of species with a wide range of volatilities. Vaporization of such fuels usually favors more volatile species throughout the whole process. Such preferential vaporization has prevailed in practical applications, such as direct-injection engine and gas turbine. To study preferential vaporization of complex fuels, a 24-component mixture proposed in our prior work was used in the present study to represent the Chinese aviation fuel, RP-3. The classical model for droplet evaporation with force convection was used to study the preferential vaporization of fuel droplet, with Effective Thermal Conductivity/Effective Diffusivity, ETC/ED models that explores various extent of liquid mixing. The effects of ambient temperature (473-973 K), pressure (1-20 bar), and force convection (0-1 $\mathrm{m} / \mathrm{s}$ ) on preferential vaporization of 24component droplet have been investigated in this work. The degree of preferential vaporization is defined as $1 / P e_{L}$. The results show that the preferential vaporization is inhibited by the increase of ambient temperature. High ambient pressure would lead to higher degree of preferential vaporization. For force convection, preferential vaporization is enhanced firstly and then inhabited by increasing intensity of force convection due to the competition between evaporation rate and liquid mass transfer.
\end{abstract}

\section{Keywords}

Preferential vaporization; 24-component droplet; ETC/ED; Lewis number; Peclet number;

\section{Introduction}

The petroleum-derived fuels like gasoline, kerosene, and diesel are major energy source of transportation vehicle, whose chemical energy is converted to thermal energy via spray combustion and then drives power machinery. Those fuels contain hundreds of species with different volatilities. It makes the lighter (higher volatile) fuel components leave the liquid phase more rapidly than the heavier (lower volatile) components, i.e. preferential vaporization, during the spray process of those multicomponent fuels. The importance of preferential vaporization for multicomponent has been presented by Senda et al. [1], which showed the vapor fuel distribution in combustion chamber of diesel engine and gasoline engine. Ra and Reitz [2] numerically studied the difference of spray behaviours between multicomponent gasoline surrogate and single component gasoline surrogate. It was found that although their overall spray penetration looks similar, the temporal-spatial distribution of fuel vapor composition is significantly different due to preferential vaporization. Besides numerical studies, some experimental measurements [3-5] using two-tracer LIF showed evidence of preferential vaporization during the spray process of multicomponent fuels. These studies demonstrated that the preferential vaporization would lead to the variation of temporal-spatial distribution of fuel composition. Furthermore, more researchers study the impacts of preferential 
vaporization on combustion behaviours of multicomponent, such as ignition [6], lean blow-out (LBO) [7], spray flame structure and propagation [8]. Due to the complexity of spray combustion, some researchers studied the effects of preferential vaporization on droplet combustion [9] or Combustion Property Targets (CPTs) during droplet evaporation [10,11], which is the sub-grid phenomena of spray combustion.

Based on studies about evaporation of multicomponent droplet, there are three factors affecting the preferential droplet vaporization of multicomponent fuel: composition, liquid mass diffusivity and surface regression rate (see Eq. (1)). Preferential vaporization stems from the volatility difference of species in multicomponent fuel, i.e. the diversity of saturated vapor pressure for each component. Besides species volatilities, the competition between species diffusivity in liquid phase and droplet surface regression rate has a great influence on the simultaneous vapor fuel composition. Makino and Law [12] suggested using the gasification Peclet number, as presented in Eq. (2), to represent the degree of such competition of multicomponent droplets.

$K_{S}(t)=-\frac{1}{2} \frac{d r_{s}^{2}}{d t}$

$P e_{L}(t)=\frac{K_{S}(t)}{D_{L}}$

Except some works $[7,9]$ above has mentioned the influence of temperature and pressure on the preferential vaporization, there are rather few studies directly investigating the effects of some dependent parameters on preferential vaporization. In present work, parametrical investigations (temperature, pressure and force convection) about preferential vaporization of multicomponent droplet are carried out. A 24-component mixture, formulated as a surrogate for Chinese aviation fuel RP-3 in prior work [13], is selected as the target fuel. To take account of force convection effect, the classical Effective Thermal Conductivity/Effective Diffusivity (ETC/ED) droplet evaporation model is utilized to simulate the evaporation of multicomponent droplet.

\section{Computational Methods}

The real complex fuel used in transport device contains hundreds of species, which is difficult to be described accurately. In our previous work, a 24-component surrogate [13] was formulated for Chinese aviation fuel RP-3 based upon the variation of functional groups during distillation experiments. Relatively successful predictions were provided using the surrogate for preferential vaporization behaviour of real complex fuel droplets. In order to efficiently simulate the droplet evaporation under force convection, the "stagnant film theory" and "effective thermal conductive/effective diffusivity" model $[14,15]$ are used in this work. In gas phase, the quasi-steady assumption and spherical symmetry are utilized in this model, which is a widely accepted assumption in droplet evaporation model. The mass evaporation rates for multicomponent droplet are presented in Eqs. (3) and (4). For these two formulas, the mixture of vapor species is treated as a separate gas. The physical properties in Eqs. (3) and (4) are calculated via the average of vapor species.

$$
\begin{aligned}
& \dot{m}_{F}=2 \pi r_{s} \frac{\lambda_{G}}{C_{p, G}} N u^{\prime} \ln \left(1+B_{T}\right) \\
& \dot{m}_{F}=2 \pi r_{S} \rho_{G} \bar{D}_{F, G} S h^{\prime} \ln \left(1+B_{M}\right)
\end{aligned}
$$

where $B_{T}$ and $B_{M}$ are Spalding heat transfer number and mass transfer number respectively. Sh' and $\mathrm{Nu}$ ' are modified Nusselt and Sherwood number, respectively, to take into account the effect of convective transport caused by relative motion between droplet and ambient gas on droplet evaporation rate. 
In liquid phase, vortex motion inside the droplet is able to promote the transport speed of energy and mass, which is treated as effective thermal and mass conductivity factors, i.e. $\chi_{T}$ and $\chi_{M}$, as shown in Eqs. (5) and (6). $\chi_{T}$ is a parameter determined by liquid Reynold number $\left(R e_{L}\right)$ and liquid Prandtl number $\left(P r_{L}\right)$, while $\chi_{M}$ depends upon liquid Reynold number $\left(R e_{L}\right)$ and liquid Schmidt number $\left(S c_{L}\right)$. Both $\chi_{T}$ and $\chi_{M}$ range from 1 to 2.72 as surveyed from the spray scenarios.

$\frac{\partial T}{\partial t}=\chi_{T} \alpha_{l}\left(\frac{\partial^{2} T}{\partial r^{2}}+\frac{2}{r} \frac{\partial T}{\partial r}\right)$

$\frac{\partial X_{i}}{\partial t}=\chi_{M} D_{i, L}\left(\frac{\partial^{2} X_{i}}{\partial r^{2}}+\frac{2}{r} \frac{\partial X_{i}}{\partial r}\right)$

At the vapor-liquid interface of droplet, the modified Raoult's law is used to describe the vaporliquid equilibrium, as shown in Eq. (7). $r_{i}$ is activity coefficient of species $i$, which is estimated using the UNIFAC method for such non-ideal mixture.

$y_{i}=x_{i} \gamma_{i} p_{v p, i}(T) / p$

The thermophysical properties in the gas phase are evaluated by the "1/3 rule" [14]. The liquid diffusion coefficient is calculated through binary liquid diffusion coefficients for dilute solvents [16]. Other thermophysical properties of liquid and vapor species can be found in [17].

Before studying the effects of dependent parameters on degree of preferential vaporization, the degree of preferential vaporization needs to be defined. The gasification Peclet number $P e_{L}$, defined as the ratio of surface regression rate to liquid mass diffusivity, is the controlling factors for gasification behaviour of multicomponent droplet. The analysis in [12] showed that large value of $P e_{L}$ favors liquid phase diffusion-limiting model (onion-skin model), while small value of $P e_{L}$ corresponds to the distillation-limiting model (distillation-like model). Furthermore, larger $P e_{L}$ means large liquid diffusion resistance, which detains heavier species in the outer layer of droplet. It would make lighter and heavier components leave liquid layer by layer, thus inhibits preferential vaporization. On the contrary, small $P e_{L}$ means liquid-phase well-mixed or small droplet evaporation rate, which renders lighter species move rapidly to droplet surface and heavier species to center of droplet. This would lead lighter species to vaporize much faster than heavier species, and hence small $P e_{L}$ enhance preferential vaporization. The analysis above between $P e_{L}$ and preferential vaporization shows negative correlation between the value of $P e_{L}$ and the degree of preferential vaporization. Therefore, the $1 / P e_{L}$ is defined as the degree of preferential vaporization in this work.

After defining the degree of preferential vaporization, the parametrical investigations with variation of ambient temperature (473-973 K), ambient pressure (1-20 bar) and force convection $(0-1 \mathrm{~m} / \mathrm{s})$ are carried out in the framework of ETC/ED droplet vaporization model introduced above. The details simulation parameters are given in Table 1.

Table 1 - Computational parameters in this work

\begin{tabular}{c|c|c|c|c|c}
\hline Variable & $d_{0}$ & $T_{d}$ & $T_{f}$ & $p$ & $U$ \\
\hline Temperature & $0.5 \mathrm{~mm}$ & $298 \mathrm{~K}$ & $473-973 \mathrm{~K}$ & $1 \mathrm{bar}$ & $0 \mathrm{~m} / \mathrm{s}$ \\
Pressure & $0.5 \mathrm{~mm}$ & $298 \mathrm{~K}$ & $773 \mathrm{~K}$ & $1-20 \mathrm{bar}$ & $0 \mathrm{~m} / \mathrm{s}$ \\
Force convection & $0.5 \mathrm{~mm}$ & $298 \mathrm{~K}$ & $773 \mathrm{~K}$ & $1 \mathrm{bar}$ & $0-1 \mathrm{~m} / \mathrm{s}$
\end{tabular}

\section{Model validation}

The model established in this work is implemented on the MATLAB platform. In order to validate the results generated by the model, some experimental data of droplet evaporation for single component and binary components fuels are referred in this section. The experimental and computational parameters are same, as shown in Table 2.

Table 2 - Computational parameters for validation cases 


\begin{tabular}{c|c|c|c|c|c|c|c}
\hline Case & $\begin{array}{c}\text { Droplet } \\
\text { composition }\end{array}$ & $d_{0}$ & $T_{d}$ & $T_{f}$ & $p$ & $U$ & Ambient gas \\
\hline $\mathrm{A}$ & $\mathrm{n}-\mathrm{C}_{7} \mathrm{H}_{16}$ & $0.5 \mathrm{~mm}$ & $298 \mathrm{~K}$ & $473 \mathrm{~K}$ & $1 \mathrm{bar}$ & $0 \mathrm{~m} / \mathrm{s}$ & $\mathrm{N}_{2}$ \\
$\mathrm{~B}$ & $\mathrm{n}-\mathrm{C}_{7} \mathrm{H}_{16}$ & $0.5 \mathrm{~mm}$ & $298 \mathrm{~K}$ & $773 \mathrm{~K}$ & $1 \mathrm{bar}$ & $0 \mathrm{~m} / \mathrm{s}$ & $\mathrm{N}_{2}$ \\
$\mathrm{C}$ & $\mathrm{n}-\mathrm{C}_{16} \mathrm{H}_{34} / \mathrm{n}-\mathrm{C}_{10} \mathrm{H}_{22}$ & $0.3 \mathrm{~mm}$ & $293.15 \mathrm{~K}$ & $1100 \mathrm{~K}$ & $1 \mathrm{bar}$ & $0.6 \mathrm{~m} / \mathrm{s}$ & $\mathrm{N}_{2}$ \\
D & $\begin{array}{c}(47 / 53 \mathrm{~mol} \%) \\
\mathrm{n}-\mathrm{C}_{16} \mathrm{H}_{34} / \mathrm{n}-\mathrm{C}_{12} \mathrm{H}_{26} \\
(47 / 53 \mathrm{~mol} \%)\end{array}$ & $0.3 \mathrm{~mm}$ & $293.15 \mathrm{~K}$ & $1100 \mathrm{~K}$ & $1 \mathrm{bar}$ & $0.6 \mathrm{~m} / \mathrm{s}$ & $\mathrm{N}_{2}$
\end{tabular}

The comparisons between simulation results and experimental data for single component droplets are given in Figure 1. The simulation results and experimental data are in acceptable agreement. Note that the experimental data from Nomura et al. [18] were obtained under microgravity. Such ideal environment promotes 1-D symmetry of droplet evaporation, from liquid to gas phase and hence provides legitimacy for comparison with our simulation, as compared to the slightly deviated data from Javed et al. [19] shown in Figure 1.

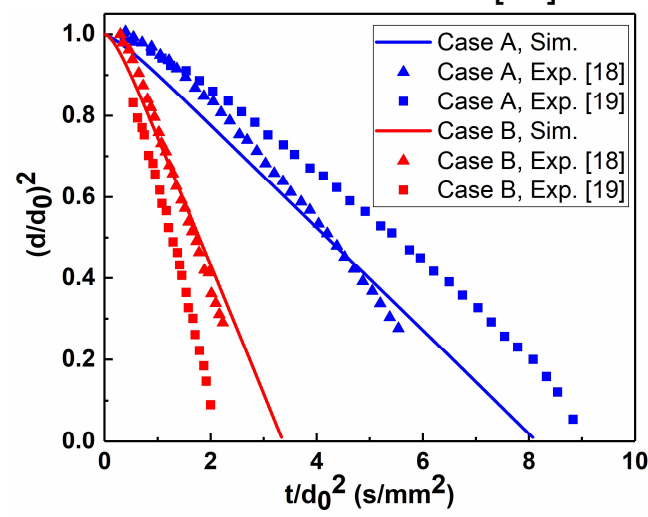

Figure 1 - Comparison of droplet regression between simulation results and experimental data $[18,19]$ for Case $A$ and Case $B$

To validate the effects of force convection on droplet gasification and liquid phase mass and heat transfer, droplet evaporation cases of binary mixtures (Case $C$ and Case $D$ in Table 2) are numerically computed. Evolution of mole fraction of light components $X_{\text {light }}$ are shown in Figure 2, with $x$-axis being $d^{2}$-Law time $(\tau)$, defined as $1-\left(d / d_{0}\right)^{2}$. The negative region of $d^{2}$ Law time, as labelled by grey region in Figure 2, is droplet heat-up period. Due to the negative correlation between liquid density and temperature, the droplet would expand at first and lager than initial droplet size. Therefore, the value of $d^{2}$-Law time would be negative firstly and then be positive. The comparisons in Figure 2 show that simulation results and experimental data in [20] have a good agreement.

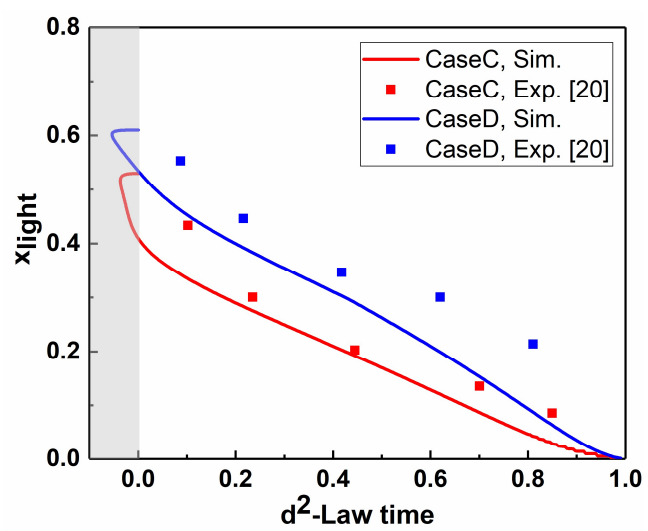

Figure 2 - Comparison of light species evolution between simulation results and experimental data [20] for Case C and Case D 


\section{Results and Discussion}

With a variation of ambient temperature (473-973 K), the degree of preferential vaporization variation is presented in Figure 3. It shows that the degree of preferential vaporization is enhanced by low temperature, which is similar to the conclusions given in [7]. The reason is that the low temperature would largely decrease the droplet evaporation rate (surface regression rate), in which the mass transfer inside the droplet has longer characteristic time. Thus, it hinders the transport of lighter species from the droplet center to droplet surface, leading higher degree of preferential vaporization. To visually demonstrate the effects of temperature on degree of preferential vaporization, the temporal-spatial distributions of components inside the droplet under different ambient temperature are presented in Figure 4. Three species $\left(n-C_{7} \mathrm{H}_{16}, n-\mathrm{C}_{10} \mathrm{H}_{22}\right.$ and $\left.n-\mathrm{C}_{16} \mathrm{H}_{34}\right)$ are selected to represent the light, medium and heavy species respectively. It shows that light species escapes the liquid phase more quickly than heavy species, i.e. preferential vaporization. Furthermore, Figure $\mathbf{4}$ also shows that the concentration of light species at low ambient temperature decreases more rapidly than that at high temperature, which indicates that the low ambient temperature enhances the degree of preferential vaporization. The consistence of results obtained from Figure 3 and Figure 4 shows the efficacy of representing degree of preferential vaporization with $1 / P e_{L}$.

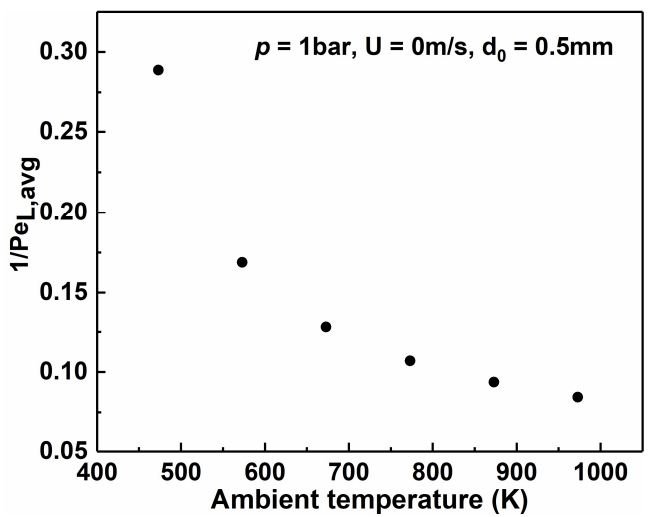

Figure 3 - Degree of preferential vaporization for jet fuel surrogate droplet operating at different ambient
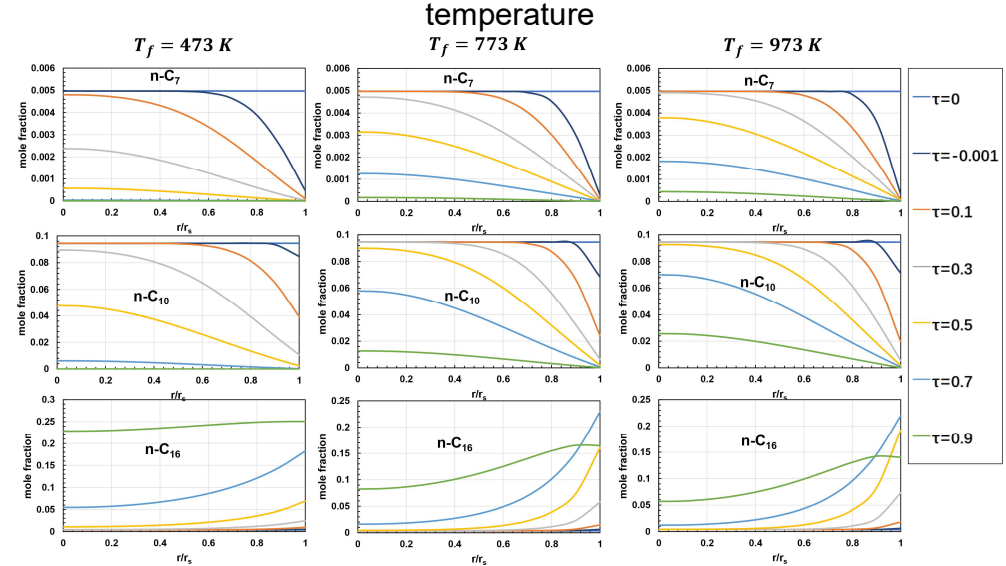

Figure 4 - Temporal-spatial variation of three species inside the droplets which operating at different ambient temperature

Figure 5 shows the effects of ambient pressure on degree of preferential vaporization of droplets under subcritical condition. With the increase of ambient pressure, the degree of preferential vaporization increase, which means that the high ambient pressure could enhance the preferential vaporization. At high ambient pressure, the boiling point of liquid mixture increases leading to a longer droplet heat-up time and slower droplet evaporation rate, which 
results in more time for light species moving from droplet centre to droplet surface. Besides, the correlation between pressure and preferential vaporization is very non-linear and tends to plateau at very high pressure. This tendency is very similar to that in droplet combustion situation, as demonstrated in [9].

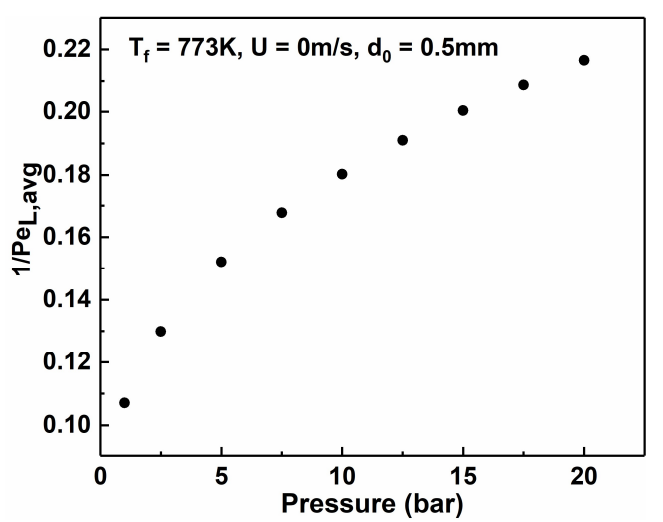

Figure 5 - Degree of preferential vaporization for jet fuel surrogate droplet operating at different ambient pressure

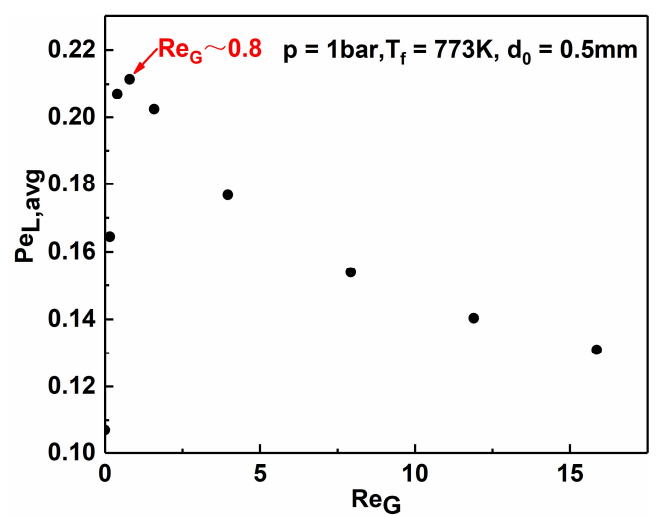

Figure 6 - Degree of preferential vaporization for jet fuel surrogate droplet operating at different force convection

The droplet vaporization under force convection is relatively complex compared with former two situations. The force convection of droplet can promote droplet evaporation rate and also mass transport speed due to vortex motion inside the droplet. The Figure 6 shows that the increase intensity of force convection would firstly increase and then decrease the degree of preferential vaporization, which is caused by the competition between droplet evaporation rate and liquid mass diffusivity. The peak of degree of preferential vaporization happens at $\operatorname{Re}_{G} \sim$ $0.8(U \sim 0.05 \mathrm{~m} / \mathrm{s})$. When $\operatorname{Re}_{G}$ less than 0.8 , the vortex motion induced by relative motion between droplet and ambient gas has large promotion on mass transfer inside the droplet. Although the evaporation rate is also enhanced by force convection, the promotion of liquid mass transfer is dominant at this stage. As a result of large enhancement of mass transfer, the degree of preferential vaporization is increased by force convection. However, due to the effects of viscosity on internal liquid circulation, the promotion of vortex motion inside the droplet by force convection has limitation. When $R_{G}$ is larger than 0.8 , the value of $\chi_{M}$ has reached its maximum value 2.72. It means that the promotion of liquid mass transport reaches its limitation with the increasing intensity of force convection. At this stage, the droplet evaporation rate is still enhanced by the force convection, which is the dominant factor to inhibit preferential vaporization. 


\section{Conclusions}

In present work, the preferential vaporization of jet fuel surrogate droplet was parametrically investigated. Firstly, with the analysis of competition between droplet surface regression rate and liquid mass diffusivity, the degree of preferential vaporization was defined as $1 / P e_{L}$. The analysis of temporal-spatial distribution of components inside the droplet shows that $1 / P e_{L}$ does well represent the degree of preferential vaporization. Besides, "film theory" and ETC/ED model was used to study the effects of ambient temperature, pressure and force convection on degree of preferential vaporization. The results show that the low ambient temperature would enhance the preferential vaporization of droplet. High ambient pressure would lead to longer droplet heat-up time and higher degree of preferential vaporization. The increasing intensity of force convection would enhance firstly and then inhibit preferential vaporization of jet fuel droplet due to the competition between droplet evaporation rate and liquid mass transfer.

\section{Acknowledgments}

This project is supported by the National Natural Science Foundation of China (NSFC) under Grant No. 51706120 and 52076117. The start-up funding from the Center for Combustion Energy, Tsinghua University as well as the Key Laboratory for Thermal Science and Power Engineering of Ministry of Education, Tsinghua University. Tsinghua University Initiative Scientific Research Program under Project No. 20193080086 are gratefully appreciated.

\section{Nomenclature}

$B_{M} \quad$ Spalding mass transfer number

$B_{T} \quad$ Spalding heat transfer number

$C_{p} \quad$ specific heat capacity $[\mathrm{J} /(\mathrm{kg} \cdot \mathrm{K})]$

$d \quad$ droplet diameter [m]

$d_{0} \quad$ initial droplet diameter [m]

$D \quad$ mass diffusivity $\left[\mathrm{m}^{2} / \mathrm{s}\right]$

$K_{s} \quad$ surface regression rate $\left[\mathrm{m}^{2} / \mathrm{s}\right]$

$\dot{m} \quad$ mass evaporation rate $[\mathrm{kg} / \mathrm{s}]$

$\mathrm{Nu} \quad$ modified Sherwood number

$p \quad$ ambient pressure [bar]

$p_{\text {sat }} \quad$ saturate vapor pressure [bar]

$P e \quad$ Peclet number

$\mathrm{Pr} \quad$ Prandtl number

$r_{s} \quad$ droplet radius [m]

Re Reynolds number

$S h^{\prime} \quad$ modified Sherwood number

$T \quad$ temperature $[\mathrm{K}]$

$T_{d} \quad$ droplet initial temperature $[\mathrm{K}]$

$T_{f} \quad$ ambient temperature $[\mathrm{K}]$

$U \quad$ incoming flow velocity $[\mathrm{m} / \mathrm{s}]$

$x \quad$ mole fraction for liquid species

$X \quad$ mass fraction for liquid species

$y \quad$ mole fraction for gas species

\section{Greek symbols}

$\alpha \quad$ thermal diffusivity $\left[\mathrm{m}^{2} / \mathrm{s}\right]$

$\gamma \quad$ activity coefficient

$\lambda$ thermal conductivity $[\mathrm{W} /(\mathrm{m} \cdot \mathrm{K})]$ 


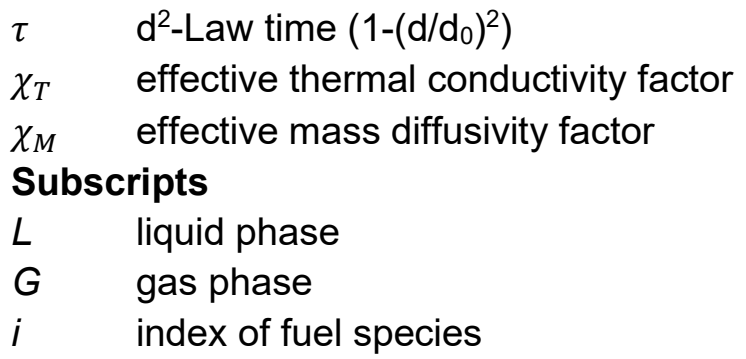

\section{References}

[1] Senda, J., Higaki, T., Sagane, Y., Fujimoto, H., Takagi, Y., Adachi, M., 2000, SAE transactions, 347-358.

[2] Ra, Y., Reitz, R. D., 2009, International Journal of Multiphase Flow, 35(2), 101-117.

[3] Itani, L. M., Bruneaux, G., Di Lella, A., Schulz, C., 2015, Proceedings of the Combustion Institute, 35(3), 2915-2922.

[4] Bardi, M., Di Lella, A., Bruneaux, G., 2019, Fuel, 239, 521-533.

[5] Kranz, P., Kaiser, S. A., 2019, Proceedings of the Combustion Institute, 37(2), 1365-1372.

[6] Stagni, A., Esclapez, L., Govindaraju, P., Cuoci, A., Faravelli, T., Ihme, M., 2017, Proceedings of the Combustion Institute, 36(2), 2483-2491.

[7] Won, S. H., Rock, N., Lim, S. J., Nates, S., Carpenter, D., Emerson, B., Lieuwen, T., Edwards, T., Dryer, F. L., 2019, Combustion and Flame, 205, 295-304.

[8] Shastry, V., Cazeres, Q., Rochette, B., Riber, E., Cuenot, B., 2020, Proceedings of the Combustion Institute, doi: 10.1016/j.proci.2020.07.090.

[9] Farouk, T. I., Won, S. H., Dryer, F. L., 2020, Proceedings of the Combustion Institute, doi: 10.1016/j.proci.2020.06.200.

[10] Cooney, A. Y., Singer, S. L., 2019, Proceedings of the Combustion Institute, 37(3), 32293236.

[11] Singer, S. L., Hayes, M. P., Cooney, A. Y., 2021, Fuel, 288, 119821.

[12] Makino, A., Law, C. K., 1988, Combustion and flame, 73(3), 331-336.

[13] Luo, L., Liu, Y. C., 2020, Proceedings of the Combustion Institute, doi: 10.1016/j.proci.2020.05.048.

[14] Abramzon, B., Sirignano, W. A., 1989, International Journal of Heat and Mass Transfer, 32(9), 1605-1618.

[15] Al Qubeissi, M., Sazhin, S. S., Crua, C., Turner, J., Heikal, M. R., 2015, Fuel, 154, 308318.

[16] Keller, P., Bader, A., Hasse, C., 2013, International Journal of Heat and Mass Transfer, 67, 1191-1207.

[17] Poling, B. E., Prausnitz, J. M., O'Connell, J. P., 2001, "Properties of gases and liquids" [18] Nomura, H., Ujiie, Y., Rath, H. J., Sato, J. I., Kono, M., 1996, Symposium (International) on Combustion, 26(1), 1267-1273.

[19] Javed, I., Baek, S. W., Waheed, K., 2013, Combustion and Flame, 160(1), 170-183.

[20] Randolph, A. L., Makino, A., Law, C. K., 1988, Symposium (International) on Combustion 21(1), 601-608. 